\title{
Three Years of Experience with the STELLA Robotic Observatory
}

\author{
Thomas Granzer, Michael Weber, and Klaus G. Strassmeier \\ Division of Telescope Control \& Robotics, Astrophysical Institute Potsdam (AIP), An der Sternwarte 16, 14482 Potsdam, Germany \\ Correspondence should be addressed to Thomas Granzer, tgranzer@aip.de
}

Received 30 June 2009; Revised 1 November 2009; Accepted 18 January 2010

Academic Editor: Joshua S. Bloom

Copyright ( $) 2010$ Thomas Granzer et al. This is an open access article distributed under the Creative Commons Attribution License, which permits unrestricted use, distribution, and reproduction in any medium, provided the original work is properly cited.

Since May 2006, the two STELLA robotic telescopes at the Izaña observatory in Tenerife, Spain, delivered an almost uninterrupted stream of scientific data. To achieve such a high level of autonomous operation, the replacement of all troubleshooting skills of a regular observer in software was required. Care must be taken on error handling issues and on robustness of the algorithms used. In the current paper, we summarize the approaches we followed in the STELLA observatory.

\section{Introduction}

STELLA is a fully autonomous robotic observatory with two $1.2 \mathrm{~m}$ az-alt telescopes located at the Izaña observatory in Tenerife, Spain $[1,2]$. STELLA-I is a classical f/8 Cassegraintype telescope, equipped with a swiveling M3 mirror to make both Nasmith foci available. STELLA-II is a highly specialized telescope with the single purpose to feed as much light as possible into an on-axis fiber. It has a single spherical mirror at f/1.95. At F1, a field corrector matches the PSF to the fiber entrance f-ratio and additionally provides a field of view of roughly 2 arcmin around the fiber. For acquisition and guiding, STELLA-II features an auxiliary, $15 \mathrm{~cm}$ refracting telescope. STELLA-I currently feeds the STELLA Echelle spectrograph (SES, see [3]), but in late 2009 the new widefield imaging photometer (WiFSIP) should be operated on this telescope. STELLA-II is currently in a testing phase.

Both telescopes are truly robotic telescopes in the sense that they autonomously react to changing weather conditions, including operation of the telescope sheltering building. The object selection is not based on a single, prescribed sequence, but is always constructed dynamically, making fast reaction easy (see Section 2). Internet connection is only necessary for data retrieval and upload of new targets to the target pool, both of which can be achieved with considerable low bandwidth. Remote observing is possible but has so far never been necessary for normal scientific observations.
In the next sections, a step-by-step receipt of how a human observer has been replaced by individual pieces of software is presented.

\section{Scheduling Observations}

Different to classic observatories, robotic observatories in general and STELLA especially, do not divide the available observing time into different time slices and dedicate these to single users. All targets are active at any time, allowing for observing campaigns that span months and, quite usually, even years. An overview on the different projects conducted at STELLA might help to mitigate the scheduling requirements. One of the key projects on the spectroscopic telescope is the investigation of stellar magnetic activity on a handful of stars. The rotational period defines the time scale at which a couple of, say, twenty observations should occur, at intervals as regular as possible. The usability of a single observation highly depends on all the other observations occurring around it. Only if a high phase coverage could be achieved, the individual observation was useful. Consequentially, the scheduling algorithm must be able to adjust the priority of such an observing project. Once started, it should be assured that it can also finish in time. If in doubt, refrain from starting the project at all. What comes to ease here is the general insensitiveness to the starting point of such programs, and the only focus lies on a proper phase coverage. 
Studying highly phase critical phenomena like the Blazhko effect in RR Lyrae star requires the observations to be timed exactly around certain, well-known phases. Here, no freedom in choosing the starting point is possible, but observations do not need to be clumped especially close together.

Observations of radial velocity curves of $\delta$ Cephei stars or extrasolar planets relax that even further. Here you aim at good phase coverage, but the spacing between individual observations does hardly matter.

Objects with a prior unknown periods should be scheduled such that no bias is introduced on subsequent period determination.

The final class of targets is those that introduce no special timing or periodicity but rather yield the highest importance if observed at optimal conditions-optimal can refer to certain astronomic conditions, like no moon-light pollution or minimum airmass (easily to predict) or certain seeing requirements (difficult to predict).

Algorithms that deal with optimized scheduling are not confined to robotic telescopes alone. Basically all robotic processes face similar problems (e.g., [4]). Very different approaches to the scheduling problem exist in the literature; those relevant to robotic telescopes will be discussed briefly.

2.1. Queue Scheduling. Queue scheduling is the simplest approach possible, but also the least flexible. A superior process, most likely a human, defines a schedule for the upcoming observing period (not too long to make reaction to bad weather periods possible, not too short to gain advantage of the automated observing process). On observation start, the queue is loaded into the system and followed task by task. A coupling with additional constraints (target must be above a certain height; target may only be observed within an hour of the scheduled time) may allow for a limited degree of flexibility, which make queue scheduling apt for single-task surveillance projects.

2.2. Critical-Path Scheduling. A scheduling algorithm that splits a single task, like an entire observing campaign, into different, atomic, subtasks with various dependencies amongst each other is known as critical-path scheduling (e.g., [5]). It can be seen as the mathematical description of a Gantt chart and is mainly used in huge construction projects, where the main focus lies on the dependency between individual subtasks. Still, the main task of scheduling the different task relative to each other remains and such criticalpath scheduling is seldom used in robotic astronomy.

2.3. Optimal Scheduling. An optimal schedule describes the particular flow of observations that allows the maximization of the scientific return measured according to a predefined metrics like shutter-open time. The high number of possible permutations of $N$ targets, $p(N) \propto N$ !, makes any algorithm a highly demanding one. In particular, unpredictable changes in environmental conditions break the optimal schedule, and a recalculation is necessary. At ground-based observatories, an optimal scheduling schema is difficult to implement due to unforeseen changes in weather conditions. The Hubble Space Telescope, however, uses a software package called SPIKE [6] that delivers an optimal schedule for 14-day periods. Attempts to use the same algorithm on groundbased facilities, for example, with the Very Large Telescope [7] or the Subaru telescope [8] have yielded some success in producing guidelines for night astronomers.

2.4. Dispatch Scheduling. The algorithm that schedules targets in real time, always according to the current (observational) conditions, is known as dispatch scheduling. From the entire pool of targets available, the algorithm calculates a per-target merit, picking then the target with the highest yield.

$$
m(t)=\sum_{i} w_{i} \cdot f_{i}(t)
$$

The summation is done over individual merits $f_{i}(t)$, with different weights $w_{i}$ for a particular merit. A balanced choice on the individual weights and merits allows for a very capable scheduling algorithm. This approach allows easy reaction on changing weather conditions and at the same time optimizes target scheduling for distinct side goals. However, it has no predictive capabilities in the sense that the currently top-rated observation will be done, regardless of an even higher yield possible in the future. Nevertheless, this approach is probably suited best for ground-based telescopes as the reaction to changing weather conditions is algorithminherent. Dispatch scheduling is thus used in many robotic telescope (e.g., [9]). On the STELLA observatory it is applied in a somewhat modified approach to compensate for the bad long-time behavior, see.

$$
m(t)=\prod_{i} v_{i} \cdot s_{i}(t) \cdot \sum_{j} w_{j} \cdot g_{j}(t) .
$$

Here, the $s_{i}$ 's, weighted with constant factors $v_{i}$, allow long-term modification of target selection (i.e., over several nights), while the $g_{j}$ 's are mainly used for short-term scheduling, that is, over the course of a given night. On STELLA, the target itself defines which merits it may use. This is similar to setting all weights on all nonspecified merits to zero but allows easier adaptation to new observing strategies: new merits may be added at any time, given that they are available at run time. Out of convenience, all $s_{i}$ 's and $g_{j}$ 's are limited within $0 \leq s_{i}(t), g_{j}(t) \leq 1$, but merits exceeding one are allowed by adjustment of the weights. Generally, three sets of weights for all $s_{i}$ 's and $g_{j}$ 's are available, reflecting the three principal priority levels: level $A$ for high-priority targets; all observations requested for a single target must be completed to allow scientific conclusions. Level $B$ indicates mid-priority targets. Observing strategy is best-effort based, with (currently) a high likeliness of all observations to be completed. The lowest priority, $C$, is designed for targets that either add some scientific value, if observed, or for low-priority targets in large surveys. As a matter of fact, targets within this priority class are mostly observed during partly clouded nights, when targets in higher priority bins fail. 


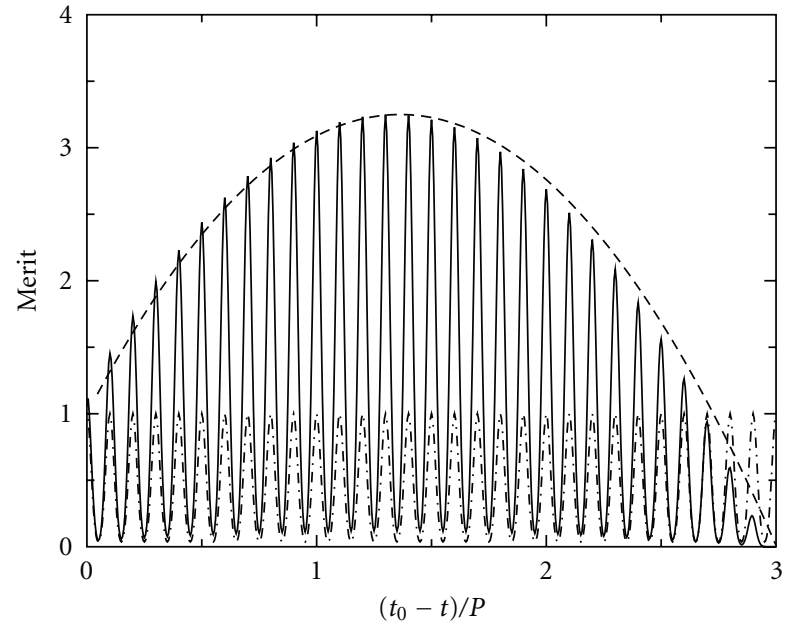

FIgURE 1: A combination of a long-term increasing and decreasing amplitude function (dashed line) with a high-frequency phaseselection wave (dotted-dashed line) to build a selection merit adequate for scheduling Doppler-imaging targets (thick line).

From the vast possibilities that the different combinations of weights and merits allow, only a few possible setups are currently implemented on STELLA. Large surveys on different targets have a pure airmass merit that peaks at one on the target's culmination and drops to zero at the apparent horizon, which lies between $5^{\circ}$ and $30^{\circ}$. Depending on the priority class, the weights are $w_{j}=1,0.67,0.33(\mathrm{~A}, \mathrm{~B}$, C). Once observed successfully, their toggle-merit $s_{i}(t)$ drops to zero. Surveys, where targets should be revisited after a certain, maybe target-depending, period have an $s_{i}$ that starts at one, drops to zero on a successful observation, and linearly regains its top value of one after the specified period.

Targets that need observation strictly at specific phases utilize a so-called phase merit, that is the sum of normalized Gaussian centered at the requested phases. Targets that provide no phase zero point are scheduled according to their first successful observation. Again the weights read as $w_{j}=1,0.67,0.33(\mathrm{~A}, \mathrm{~B}, \mathrm{C})$. In the basic form, where only strict phasing, but not the evolved time span matters, the single $s_{i}$ is constructed such that it starts at $1 / N$, where $N$ is the number of phases requested. As the number $n$ of phases successfully observed increases, $s_{i}$ follows $s_{i}=(1+n) / N$. The accompanying weight $v_{i}$ equals $v_{i}=N$, leading to a gentle increase in total merit from one to $N$ once the target has been started.

The most ambitious scheduling is done for objects that need a couple of phases observed within a few periods. At STELLA Doppler-imaging targets, these periods are in the range of several days, leaving you 2-3 weeks to complete a target. The merit is constructed in the following way. We start with an $s_{1}$ that peaks around the opposition of the target, mathematically a normalized Gaussian with an FWHM of three periods. Once such a target is successfully observed for the first time, a second $s_{2}$ gets activated, which is a parabola fit through points $1 / v_{2}$, the reciprocal of the weight at the starting time and through zero after some multiple of the

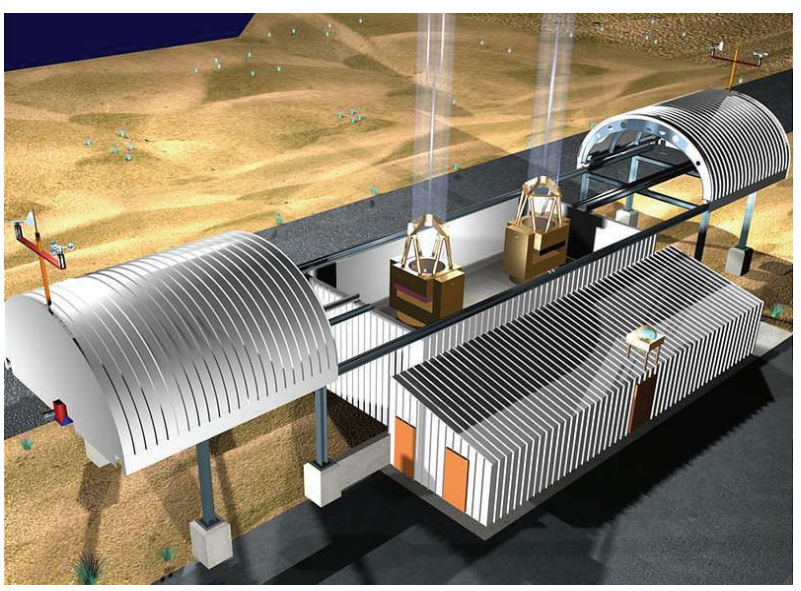

FIgure 2: The layout of the STELLA building with the two rolloff roof halves. The building can be closed independently of the telescopes' positions. This allows for faster closing times, and, even more importantly, it allows for shutdown also in case of telescope movement failures.

period, default after $t=3 P$. Additionally, a time until the maximum is reached can be specified. In addition with the beforementioned phase merit, a complex merit functions like the one depicted in Figure 1 are reached. Some more details can be found in [10].

\section{How to Carry out a Robotic Observation}

When a target has been selected by the scheduling process, all the individual subtasks for that particular target have to be carried out-in parallel whenever possible, strictly sequential if dependencies between the subtasks exist. Parallelizing many tasks can save valuable observing time, on STELLA, read-out of the scientific CCD takes place while the telescope already slews to the next target. In pretty exactly half of the cases, the next target is already acquired and closed-loop guiding has commenced, when the read-out finishes.

STELLA houses a spectroscopic and an imaging telescope, thus the individual subtasks for a single observation differ quite substantially, nevertheless the general idea of splitting an observation into subtasks with the possibility to execute them in parallel or sequentially stays the same. In STELLA, we implemented a generic sequencing schema, which is described in detail in [11]. There we also explain how targets can define their own observing sequence and how a template sequence is constructed. In this paper, however, we want to focus more on the individual subtasks and their implementation, particularly pointing out that the solutions have been chosen for robustness rather than high accuracy. In the description, we follow the principle time line of an astronomic observation, starting by judging the overall weather situation, followed by pointing and focusing of the telescope, then acquiring of a target, followed by closed-loop guiding during the scientific exposure. These sections apply particularly to the fiber-fed instrument Stella-I. Tasks only 


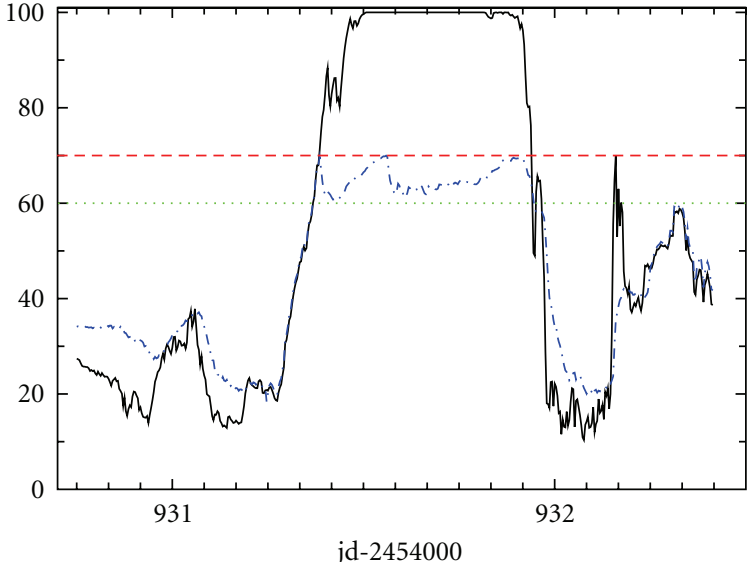

jd-2454000

(a)

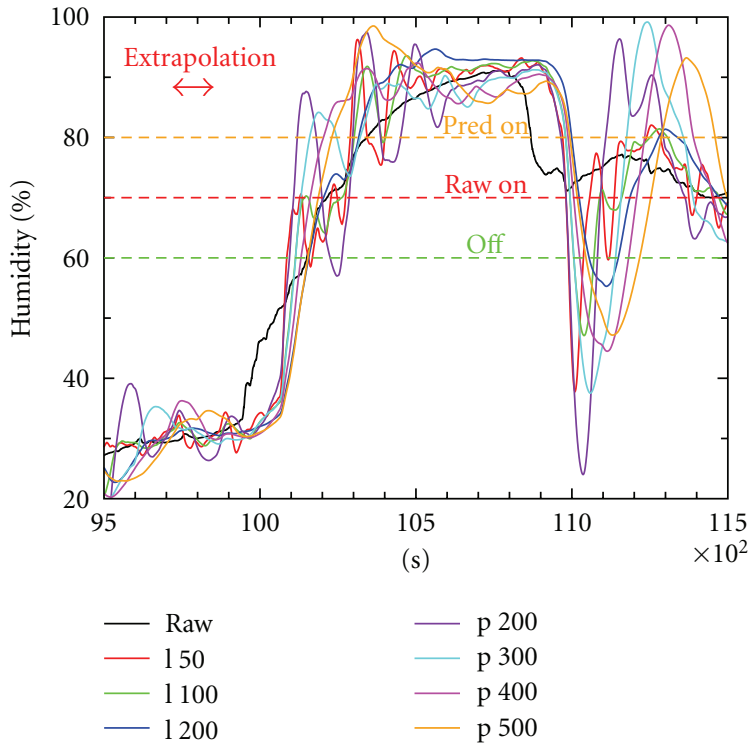

(b)

Figure 3: (a) The response of the environment system to the outside humidity (thick line). When a level of $70 \%$ on the rising edge is passed, the roofs are closed (note that the bay humidity, dot-dashed, stays roughly constant thereafter. Reopening of the building is allowed, once the humidity has dropped below $60 \%$ and remained at that level for at least two hours. (b) Prediction of inversion layer breakdowns. The thick line is the measured relative humidity. The 100-second extrapolations of various extrapolators are superimposed onto the true measurement. The true development of the humidity could be foreseen by all of the extrapolators. Note the delayed onset of the humidity rise of the extrapolated to the true values. The extrapolation could not predict that a sharp rise will start to occur, but the goal to predict the height of the humidity rise is well matched.

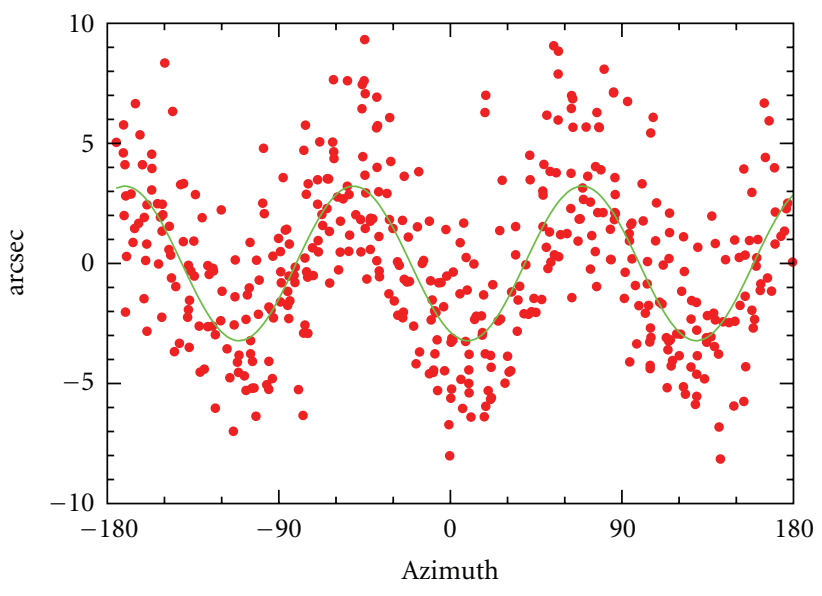

- alt (res.)

FIgURE 4: The RMS of the elevation pointings after a classical pointing model has been applied compared to the true offsets. The RMS varies at an amplitude of $\approx 3^{\prime \prime}$, at a wavenumber $k=3$, reminiscent of the tripod mounting of the telescope.

required for the imaging telescopes Stella-II follow in the next Section 4.

3.1. Protecting the Telescope in Harsh Weather Conditions. Protecting the telescope from the outside during bad weather conditions is one of the task that an autonomous observatory has to fulfill with the highest possible reliability. Needless to say that it is better to lose some observing time at unstable weather conditions than to risk damage to the telescope or the instrumentation due to high humidity, rain, high wind, or, particularly cumbersome in Tenerife, dust. The STELLA observatory is laid out as a building with a roll-off roof with two, elliptically shaped roof halves driven by crane motors. Opening and closing of the roof is possible in any position of the two telescopes; see Figure 2. As an additional security mechanism, a simple watchdog system that automatically closes the roof in case of computer crashes is used. Systems where the telescope has to be moved to a certain position before the closing of the roof can commence should be avoided whenever possible, as it leaves the instruments in an unprotected state if the telescope, for whatever reason, cannot be turned.

In standard, that is, unattended operation mode, the meteorological readings of two independent weather stations decide on the opening or closing of the roof. All sensors considered critical (precipitation, humidity, temperature, and wind speed) are available at either station, with the redundancy allowing for operation of the observatory even in case of failure of one of the stations. The building control acts completely independent from all other systems and had been installed even before the telescopes was put in place. In almost eight years of building operation, it never failed to protect the telescope.

The decision-making process for the open/close roof process relies on the current reading of the four critical 


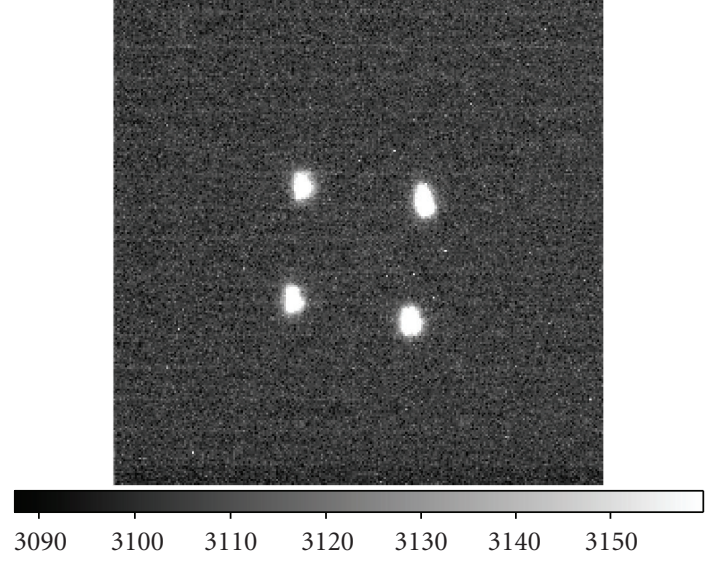

(a)

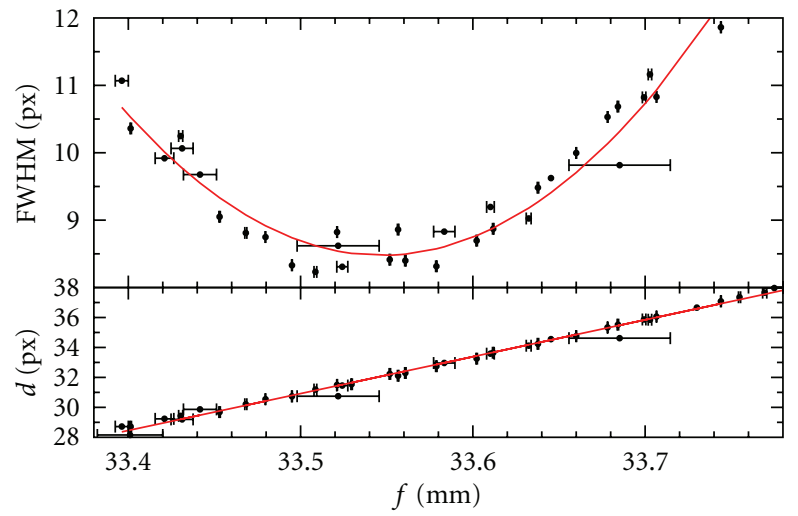

(b)

FIgURE 5: (a) The image of a single star with the focus pyramid introduced into the beam. Possible errors in the center-of-gravity determination of the four image centers do not hamper the focussing accuracy due to the high sensitivity of the subimages' distances on the focus position. (b) The calibration curve used to convert the pyramid's subimage distances to the focus applied. (a) shows the measured FWHM of a star without the pyramid in the optical beam as a function of focus position (distance of the secondary mirror to M1). (b) shows the average distance of the four subimages in pixel with the pyramid inside the optical beam. This calibration has been done at a very early time in the commissioning of the telescope, when the telescope control system was still unstable in attaining certain focus positions. This is visible as the sometimes huge error bars in the focus position.

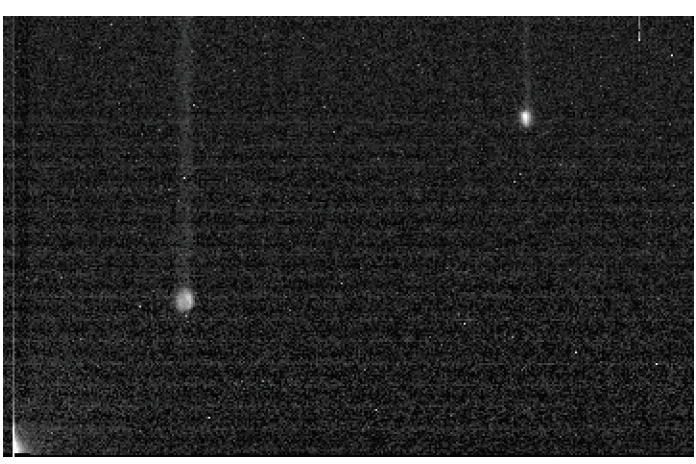

(a)

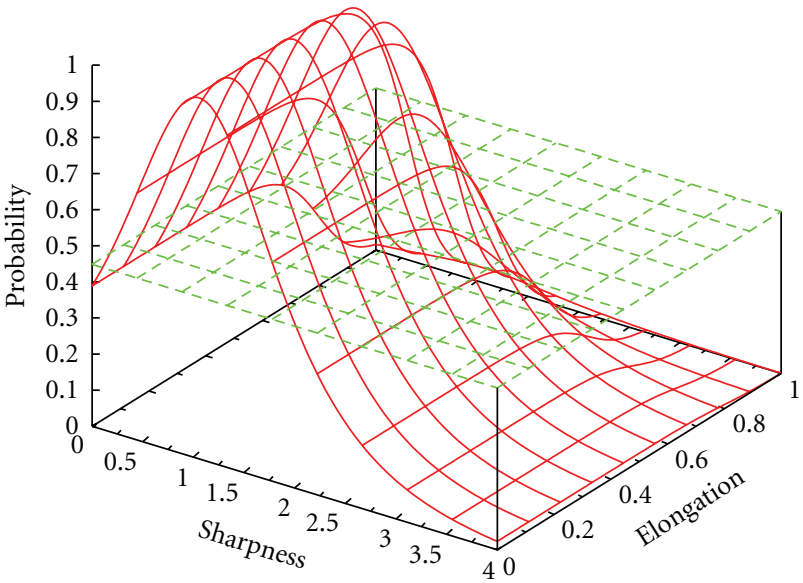

(b)

FIgURE 6: (a) A typical first image during the acquire phase. The image of the star through the beam splitter is the brighter spot to the left the fainter image to the right is the image from the mirror around the fiber entrance. The read-out stripes result from the shutter-less design. (b) The probability function used for identifying stars from their measured image elongation ( $y$, to the back) and sharpness ( $x$, to the right). The green, constant level at a probability of 0.446 is the threshold above which stars are identified. The two-dimensional function in red was derived by a manual training of the acquire system on 100 different images.

sensors, as well as on the history of their measures. A solar-height sensor is a purely calculated sensor but enters just like another critical sensor. Combining two or more sensors in the decision making turned out to be not necessary.

Most of the critical sensors act as Schmidt-triggers; they toggle their weather state on two values, depending on the general direction of the past measures. Toggling from one state into the other is additionally delayed by a sensordependant retard time, during that the sensor's reading must stay in the new state, otherwise toggling does not occur.
Typically, this retard time is two seconds or four read cycles if toggling to the bad weather state-its main purpose is to filter out erroneous readings of a sensor. Toggling to the good weather state is more depending on the site characteristics. For Izaña, humidity toggles to bad at $70 \%$ the clear toggle is set at $60 \%$ Wind speed toggles at $20 \mathrm{~m} / \mathrm{s}$ and $10 \mathrm{~m} / \mathrm{s}$, respectively, temperature at $-2^{\circ} \mathrm{C}$ and $+1^{\circ} \mathrm{C}$. Precipitation has just an on/off state, thus zero is considered no rain, one is considered raining. A brightness sensor toggles at $3000 \mathrm{~lx}$ and $10000 \mathrm{~lx}$. It is mainly a guard against wrong-posed solar height calculations. 
We apply a retard time of two hours for a humidity event, four hours for a rain event, and twenty minutes for a high wind-gust event. This principle is depicted in Figure 3(a), for reaction on the humidity.

Early on, the exposed location of the Tenerife site, just above the inversion layer, demanded for the capabilities to predict inversion layer breakdowns. For that, the course of the humidity is examined in more detail and a prognosis for the next 100 seconds-the closing time of the roofsis derived from it; see Figure 3(b). Different time bases and either linear or parabolic extrapolation are used for the nearfuture humidity development. Only if at least six out of seven extrapolators predict a humidity above $80 \%$-compared to the normal toggle of $70 \%$ - the roofs close.

3.2. Pointing the Telescope. For a reliable object acquisition, a good initial pointing of the telescope is desirable. For that, a stable mount is inevitable. To compensate for the small optical and mechanical misalignments of even the most precise telescope mounts, a mathematical model known as the pointing model is used to bring the initial pointing errors down to less than a few arc seconds. STELLA uses the classical pointing model, which describes only effects of misalignment of an otherwise perfect mount. The corrections to the azimuth $(A)$ and the elevation $(E)$, namely, $\Delta A$ and $\Delta E$, are modeled according to (3). $A_{0}$ and $E_{0}$ are constant offsets; the two angles $A_{N}$ and $A_{E}$ describe the tilt of the telescope's azimuth axis to the true vertical in the northern and eastern direction, respectively. $N_{\mathrm{PAE}}$ describes the nonperpendicularity between the telescope's altitude and azimuth axis. $B_{\mathrm{NP}}$ is the nonperpendicularity between the telescope's altitude axis and the optical axis, while $T_{F}$ is the tube flexure

$$
\begin{aligned}
\Delta A= & A_{0}-B_{\mathrm{N} P} \sec E+N_{\mathrm{PAE}} \tan E+A_{N} \cos A \tan E \\
& +A_{E} \sin A \tan E, \\
\Delta E= & E_{0}-A_{N} \sin A+A_{E} \cos A+T_{F} \cos E .
\end{aligned}
$$

Ignoring the constant offset $A_{0}$ and $E_{0}$, the highest absolute values are for $T_{F}=49^{\prime \prime}$ and for $B_{\mathrm{NP}}$ and $N_{\mathrm{PAE}}$ at $B_{\mathrm{NP}}=$ $28^{\prime \prime}$ and $N_{\mathrm{PAE}}=32^{\prime \prime}$, but note also the discussion on pointing models in [12]. One specific effect of the mount of the STELLA telescopes is depicted in Figure 4; the tripodmooring of the telescope mount has its resemblance in a $120^{\circ}$, wavelength modulated RMS in the elevation pointing corrections.

3.3. Focusing with a Focus Pyramid. Different solutions for focusing a telescope exist. On Stella-I, we tried to aim at a fast and reliable procedure. We decided to equip the telescope with the so-called focus pyramid, which can be rotated into the optical beam. It splits the light of a point-source, that is, of stars, into four individual subimages, whose distances are a direct measure of the focus; see Figure 5. Once calibrated, a single measurement of the subimages' distances suffices to determine the focus.
3.4. Acquiring the Target. Currently, Stella-I is fiber-feeding an Echelle spectrograph [3]. Thus target acquisition must be done at a precision of less than an arc second. During integration, an adapter unit allows permanent position control down to subarc second levels. This adapter unit hosts a gray beam splitter that diverts $4 \%$ of the target light onto a guiding camera. The region just around the fiber entrance is imaged with a mirror onto the same guiding camera, leading to a second image at a varying offset from the direct beam splitter image; see Figure 6(a). The guiding camera has a shutter-less design to minimize the number of moving parts in the system. The read-out strips inherent to such a design are dealt with in the acquisition software. For details of the optical layout of the acquire unit, please refer to [1].

Once the telescope has been moved to the target position, an image of the entire guider's field of view $(2.1 \times 1.5 \mathrm{arcmin})$ is taken; see Figure 6(a). The image is bias-subtracted and a truncated Gaussian filter is applied to it. Similar to DAOfind [13], the resulting image is used to detect star candidates. Their sharpness and their elongation are validated against a two-dimensional probability function, shown in Figure 6(b). If no stars are found, the initial acquire is repeated up to five times with gradually increased exposure times. Once an ensemble of stars has been identified, their positions are matched to the UCAC2 [14] catalog and the shift to the current telescope's position is determined. After the first successful shift, this procedure is repeated using a much smaller window centered around the mirrored fiber entrance to finally reach an offset of less than $1.5^{\prime \prime}$. This relatively large offset is necessary because image motion induced by atmospheric turbulence at short exposure times can amount to an RMS in the target position of up to $0.5^{\prime \prime}$.

3.5. Closed-Loop Guiding on the Target Star. Once the star has been successfully acquired, the closed-loop guiding system starts. Its only aim is to keep the direct image of the target star as close to the mirrored fiber entrance position as possible. The target star's brightness defines the exposure time during the guiding phase; it is adjusted to get a stellar signal at a $S / N$ ratio of $S / N \approx 5$. The read-out time of the guider window limits the exposure time to 500 milliseconds. Typically, 3000 guider frames are taken during a one-hour integration. A combined frame of all individual guider frames can be seen in Figure 7. This combined frame is also used to measure the light loss at the fiber entrance by aperture photometry of the two stellar images.

Due to atmospheric image motion, wind shake, and intrinsic telescope oscillations, not every offset measured should be applied directly to the telescope. In STELLA, a split approach is used. First, five offsets are averaged. If the average offset is less than the standard deviation, or if it is less than the expected image motion induced by the atmosphere, it is set to zero. This average offset is split into azimuth and altitude and is fed into two PID controls (from Proportional-Integral-Derivative; for an introduction to PID refer to [15]). The output of the PIDs is then applied to the telescope. During commissioning, three distinct weather situations have been identified, each triggering the use of 


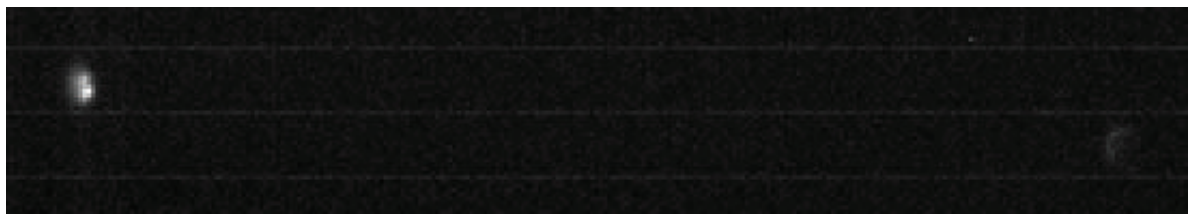

Figure 7: The combined image of $\approx 1200$ guiding frames on $51 \mathrm{Peg}$, total exposure time 20 minutes. The bright image to the left is the position of the star as imaged through the beam splitter the fainter image to the right is the light spilled over the fiber entrance and reflected back onto the guider camera. Guiding is done exclusively at the brighter image. Aperture photometry of the combined frame is used to measure the amount of light lost, here $32 \%$.

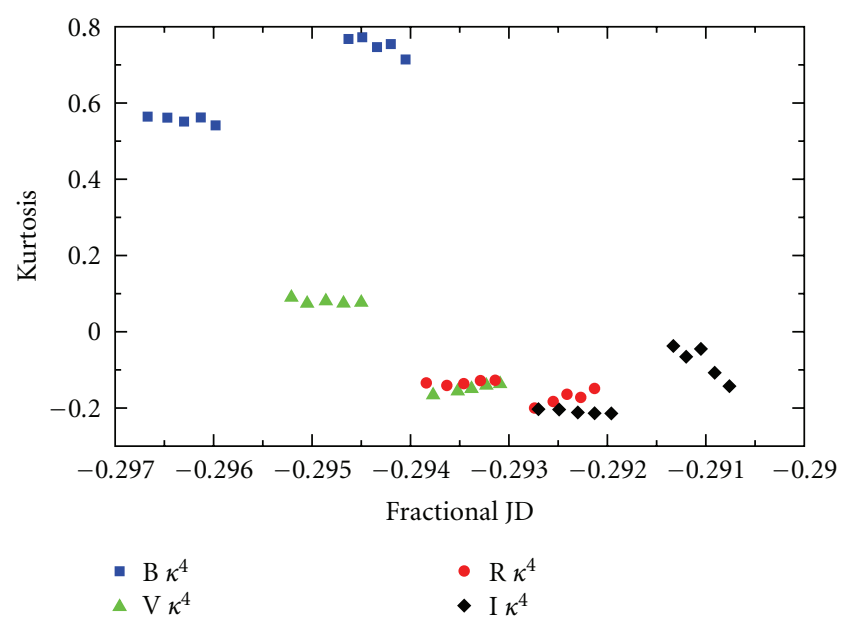

FIGURE 8: The kurtosis of the ADU statistics versus fractional JD of sky flats taken at the Mont Sec observatory during dusk twilight, five days apart. The different symbols refer to the different JohnsonCousins filters B, V, R, and I. Within each night, the kurtosis stays remarkably constant, allowing for immediate identifications of flats affected by cosmics or clouds. The kurtosis offset in the blue filters visible between the two nights is probably caused by different efficiency in the light-straying process in Earths atmosphere.

a different parameter set. In normal mode, a proportional term of $P=0.3$ for altitude and $P=0.4$ for azimuth is used. The integral constant I equals $I=0$, and for the derivative term $\mathrm{D}, D_{\mathrm{az}}=0.05$ and $D_{\text {alt }}=0.02$ are used. The badseeing mode, effective when the seeing is worse than $1.5^{\prime \prime}$, has a reduced $P_{\mathrm{az}}=0.2, P_{\text {alt }}=0.15$, and no $\mathrm{D}$ or I term. In high-wind mode for wind speeds $v>7 \mathrm{~m} / \mathrm{s}$, the number of individual offsets averaged increases from five to 20, therefore acting on a much slower time scale. $P$ is thus relatively high, $P_{\mathrm{az}}=0.3, P_{\mathrm{alt}}=0.25$, and a low I term of $I=0.05$ is introduced. $D$ stays at zero.

\section{The Imaging Telescope STELLA-II}

Different to the spectroscopic telescope, commissioning on the imaging telescope is just beginning. What follows are first results and insights gained, but far from being backed by years-long experience as in the spectroscopic case. Nevertheless, we want to address a few problems and their possible solutions in high-precision robotic photometry. For a more detailed description on the capabilities of the

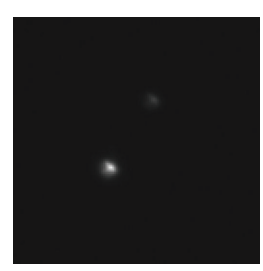

(a)

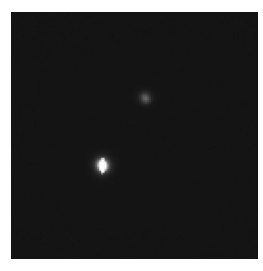

(b)

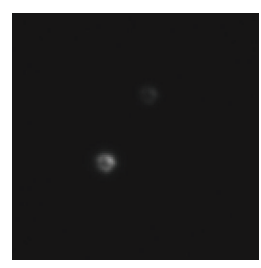

(c)

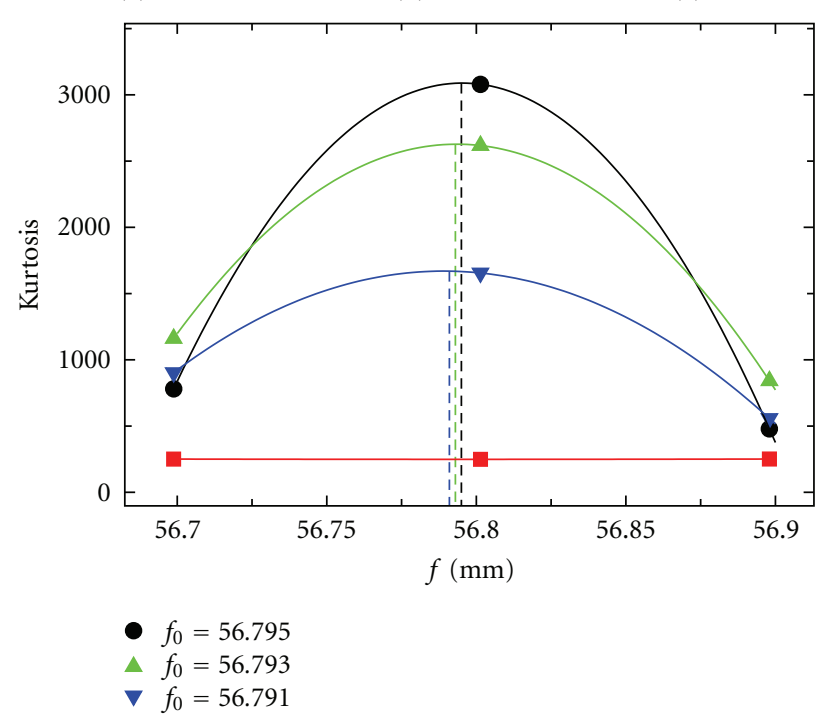

(d)

FIgURE 9: The kurtosis of the ADU statistics on three images taken with WiFSIP during precommissioning on Robotel, at the Astrophysical Institute in Potsdam. All three quadrants of the imaging CCD with bright stars in them show a peaking kurtosis close to the best focus position. The one flat line is the kurtosis in quadrant 1 , which was essentially free of stars. The small inserts on top are tiny windows around two bright stars in the field at the different focus positions.

Wide Field STELLA Imaging Photometer (WiFSIP), see, for example, [16].

4.1. Ensuring High-Quality Flat Fielding. We do not intend to equip the STELLA building with a flat-fielding screen. The time of twilights will be used to obtain sky flats. The total number of 21 filters available in WiFSIP makes it impossible to obtain twilight flats on all filters each night. The individual filter will be grouped together according to their filter system 


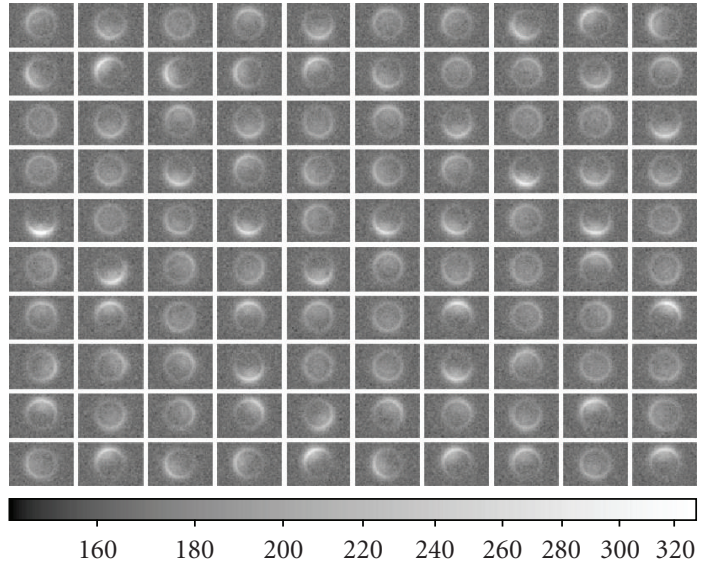

(a)

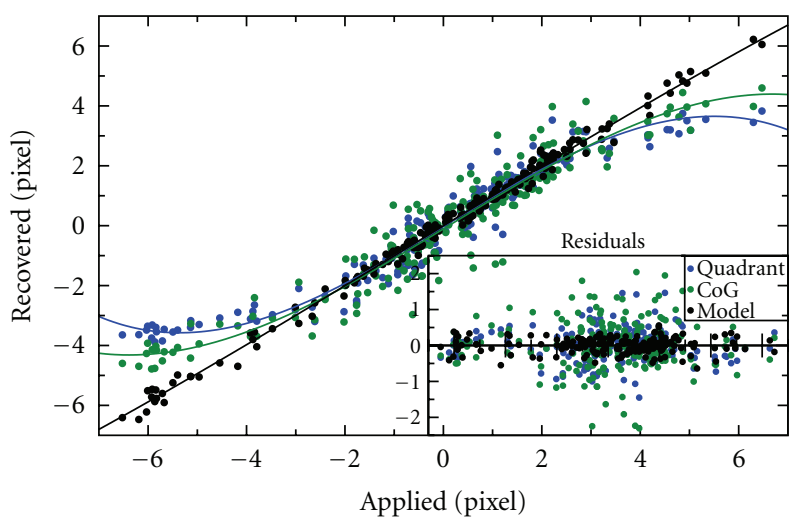

- Quadrant ( $\mathrm{rms}=0.453 \mathrm{px})$

- Center of gravity $(\mathrm{rms}=0.694 \mathrm{px})$

- Model $(\mathrm{rms}=0.206 \mathrm{px})$

(b)

Figure 10: (a) Sequence of artificial images of an $R=8^{m}$ star on the fiber viewing Unibrain 520b camera on Stella-II, exposure time set to 1 second. Pixel scale is $0.13 \mathrm{arcsec} /$ pixel the diameter of the pinhole is $2.8 \mathrm{arc} \mathrm{seconds}$. The seeing was set to 1 arcsec. (b) Recovering of the shift of the center of the star relative to the center of the pinhole, for the image sequence to the left. Center of gravity uses first image moments the quadrant method compares the ADU sum in the left-to-right and up-to-down image segments. These two methods do not deliver a true shift, but only a shift direction. For clarity, the direction has been scaled to match the true offset. Direct modeling of the star plus pinhole recovers the true shift and allows to estimate the light loss and seeing, but requires relatively high $S / N$ levels.

(Sloan, Strømgren, Johnson-Cousins), and flats in single, but entire groups should be obtained in a single twilight. Two main drivers will define which group of flats will be chosen, the time passed since the last calibration of the filter group, and the likeliness of science observations with filters of that group in the upcoming night.

Following the text book, we start with the filter of the shortest central wavelength on dusk twilight and with the red filter on dawn twilight. Small-window test exposures are taken in rapid succession until the average ADU level in the read-out window suggests an exposure time in the allowed range -0.2 to 5 seconds. The Bonn shutter of the instrument allows for such very short exposures. On OmegaCam, a similar shutter is reported by [17] to deliver equally exposed images at exposure times down to 0.1 second.

To minimize the effect of sky-brightness gradients, the telescope is pointed to the anti solar azimuth and five degrees away from the zenith. This region in the sky is the one with the lowest, sometimes even vanishing sky brightness gradient; see [18]. Additionally, the camera will be rotated by $180^{\circ}$ after half of the individual exposures. Exposure times are gradually changed to keep the average exposure level as constant as possible.

The quality of the flat-field image is assessed via ADU statistics, with particular focus on higher-order moments. In Figure 8, the kurtosis of the ADUs for five sky flats, taken in Johnson-Cousins BVRI at two different days, is plotted. The flats are from the Mont Sec robotic observatory [19], separated by five days. Though the average ADU level dropped from almost 50000 to below 30000, the kurtosis, or, equivalent the fourth central moment, stayed remarkably constant. Cosmics show up in the kurtosis as high values. Higher moments then the kurtosis works even better in detecting cosmics but also probes the arithmetic accuracy of the CPU.

4.2. Focusing Using Statistical Moments. On the imaging telescope, no auxiliary focusing unit like a focus pyramid will be present. The plan is to use a twofold approach. Depending on the temperature of the telescope structure, a focus position will be estimated. At this estimated focus position together with offsets at $\pm 0.05 \mathrm{~mm}$, three images of a field close to the celestial North pole (or maybe of Polaris itself, this will be decided during on-site commissioning) will be taken, and the kurtosis of the images will be analyzed. Figure 9 shows the anticipated procedure; the focus will be found at the point maximizing the kurtosis of the image. We hope that this procedure is superior to directly minimizing the FWHM of stellar images.

\section{Future Plans}

In spring 2010, the spectrograph-feeding fiber will be moved from Stella-I to the second STELLA telescope. The light coupling occurs at $\mathrm{F} 1$, leaving no place for a large acquire field. Acquiring will be done using a piggy-back auxiliary telescope, a $15 \mathrm{~cm}$ refracting telescope equipped with a shutter-less, uncooled guiding camera. Again, details on the optical layout can be found in [2].

5.1. Guiding on Spilled-over Light. The main acquiring and guiding system will be built around the current acquire and guiding logic, but with one additional complexity. Due to unavoidable, relative bending of the auxiliary telescope to the main telescope, guiding with the auxiliary telescope alone 
will not be sufficient. Additionally, we will use the light spilled over on the fiber entrance as a secondary guiding signal. This light will be caught on a fast read-out Unibrain $520 \mathrm{~b}$ video camera. This camera comes with an electronic shutter, allowing for exposure times lower than $1 \mathrm{~ms}$ and up to 65 seconds. Furthermore, the gain of the CCD can be adjusted quasicontinuously from 2.8 to 0.11 . This extreme range will suffice to guide on stars as bright as zeroth magnitude down to, say, $12^{m}$. First tests of the camera on Robotel allowed to estimate the quantum efficiency (QE) of the entire system to $\mathrm{QE} \approx 0.15$. Using artificial images, see Figure 10, we tested three algorithms to recover the shift of the stellar image with respect to the center of the pinhole: Center of gravity (CoG, based on image moments), quadrant weights (QWs), where the total ADUs in the left-to-right and up-to-down sectors have been compared to give a correction direction, and direct modelling of the star plus pinhole. Only direct modelling returns a true shift; CoG and QW can only deliver the principal direction of the correction. As guiding is done in a PID environment anyhow, this shortcoming does hardly matter as it is compensated by a proper choice of the proportional term.

The results of all three methods are shown in Figure 10(b). Basically all of the three methods could be used to retrieve the original shift. The higher robustness favors CoG and QW over direct modelling, while the latter delivers as a side product also information on light loss and stellar FWHM. Consequentially, we will use CoG during the guiding process and model the star plus pinhole on the combined frame to extract the additional information on light loss and seeing.

\section{References}

[1] K. G. Strassmeier, T. Granzer, M. Weber, et al., "The STELLA robotic observatory," Astronomische Nachrichten, vol. 325, no. 6-8, pp. 527-532, 2004.

[2] K. G. Strassmeier, "STELLA: two new robotic telescopes for binary-star research," Astrophysics and Space Science, vol. 304, no. 1-4, pp. 397-400, 2006.

[3] M. Weber, T. Granzer, K. G. Strassmeier, and M. Woche, "The STELLA robotic observatory: first two years of highresolution spectroscopy," in Advanced Software and Control for Astronomy II, A. Bridger and N. M. Radziwill, Eds., vol. 7019 of Proceedings of SPIE, p. 70190L, 2008.

[4] M. Pinedo, Scheduling: Theory, Algorithms, and Systems, Prentice-Hall, Upper Saddle River, NJ, USA, 1995.

[5] C. Hendrikson and T. Au, Project Management for Construction: Fundamental Concepts for Owners, Engineers, Architects, and Builders, Prentice-Hall, 1989.

[6] M. Giuliano, "Achieving stable observing schedules in an unstable world," in Astronomical Data Analysis Software and Systems VII, R. Albrecht, R. N. Hook, and H. A. Bushouse, Eds., vol. 145 of ASP Conference Series, p. 271, 1998.

[7] A. M. Chavan, G. Giannone, D. Silva, T. Krueger, and G. Miller, "Nightly scheduling of ESOs very large telescope," in Astronomical Data Analysis Software and Systems VII, R. Albrecht, R. N. Hook, and H. A. Bushouse, Eds., vol. 145 of ASP Conference Series, p. 255, 1998.
[8] T. Sasaki, G. Kosugi, R. Hawkins, J. A. Kawai, and T. Kusumoto, "Observation scheduling tools for Subaru telescope," in Optimizing Scientific Return for Astronomy through Information Technologies, P. J. Quinn and A. Bridger, Eds., vol. 5493 of Proceedings of SPIE, pp. 367-372, 2004.

[9] S. N. Fraser, "Scheduling for Robonet-1 homogenous telescope network," Astronomische Nachrichten, vol. 327, no. 8, pp. 779-782, 2006.

[10] T. Granzer, "What makes an automated telescope robotic?" Astronomische Nachrichten, vol. 325, no. 6-8, pp. 513-518, 2004.

[11] T. Granzer, "Generic control of robotic telescopes," in Advanced Software, Control, and Communication Systems for Astronomy, L. Hilton and G. Raffi, Eds., vol. 5496 of Proceedings of SPIE, pp. 667-678, 2004.

[12] K. G. Strassmeier, K. Agabi, L. Agnoletto, et al., "Telescope and instrument robotization at Dome C," Astronomische Nachrichten, vol. 328, no. 6, pp. 451-474, 2007.

[13] P. B. Stetson, "DAOPHOT-a computer program for crowded-field stellar photometry," Publications of the Astronomical Society of the Pacific, vol. 99, p. 191, 1987.

[14] N. Zacharias, S. E. Urban, M. I. Zacharias, et al., "The second US naval observatory CCD astrograph catalog (UCAC2)," Astronomical Journal, vol. 127, no. 5, pp. 3043-3059, 2004.

[15] A. Visioli, Practical PID Control, Springer, Berlin, Germany, 2006.

[16] K. G. Strassmeier, T. Granzer, M. Weber, et al., “The STELLA robotic observatory on Tenerife," Advances in Astronomy, vol. 2010, Article ID 970306, 11 pages, 2010.

[17] K. Reif, G. Klink, P. Müller, and H. Poschmann, "The OmegaCam shutter," in Scientific Detectors for Astronomy, P. Amico, J. W. Beletic, and J. E. Beletic, Eds., Astrophysics and Space Sciences Library, 2004.

[18] F. R. Chromey and D. A. Hasselbacher, "The flat sky: calibration and background uniformity in wide-field astronomical images," Publications of the Astronomical Society of the Pacific, vol. 108, no. 728, pp. 944-949, 1996.

[19] J. Colomé, D. Fernández, J. Isern, et al., "Robotic design of the Montsec astronomical observatory," Astronomische Nachrichten, vol. 325, no. 6-8, p. 658, 2004. 

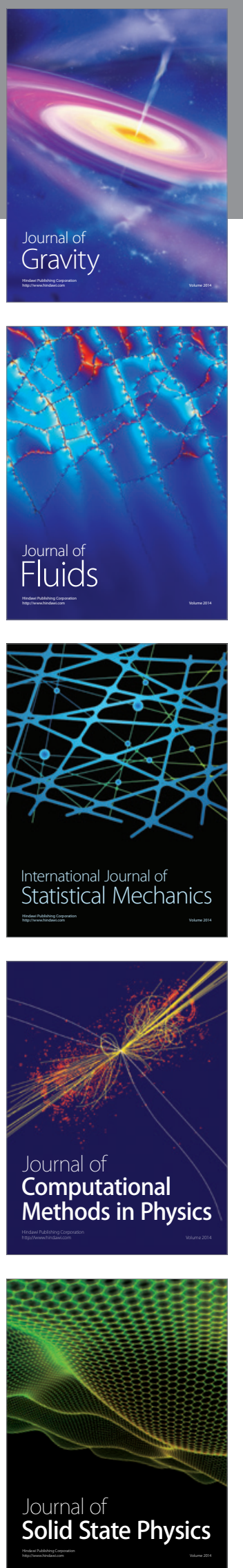

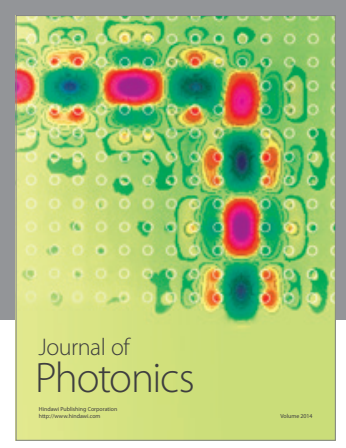

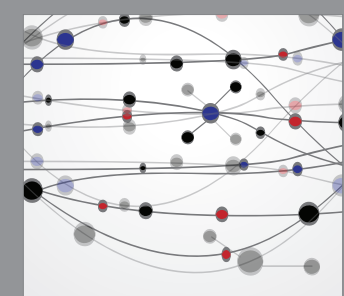

The Scientific World Journal
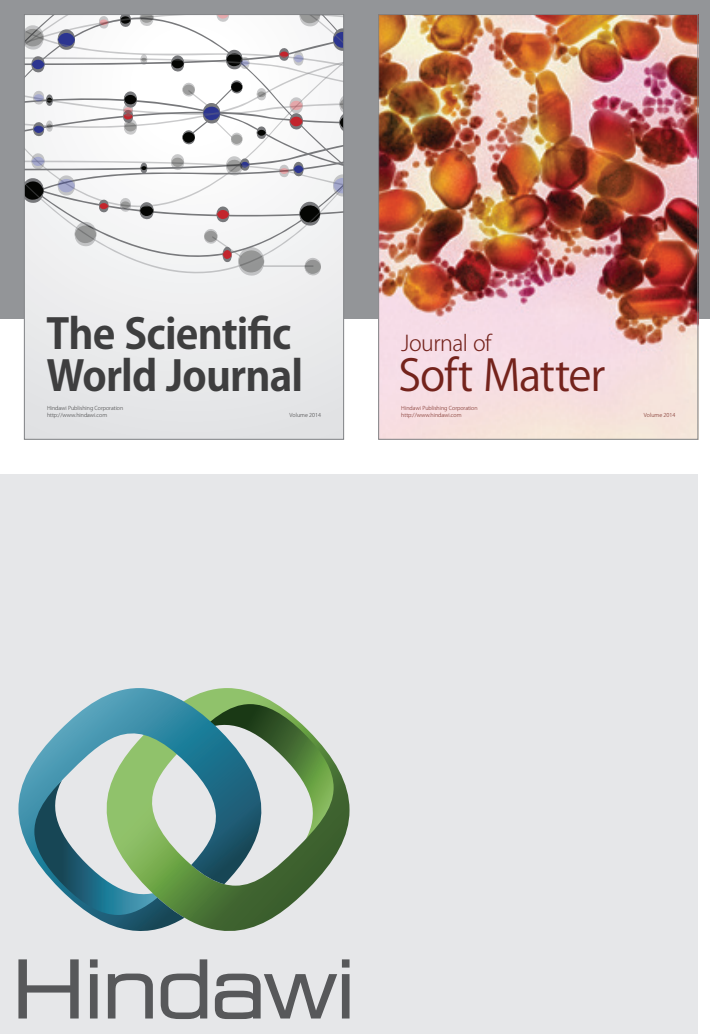

Submit your manuscripts at

http://www.hindawi.com
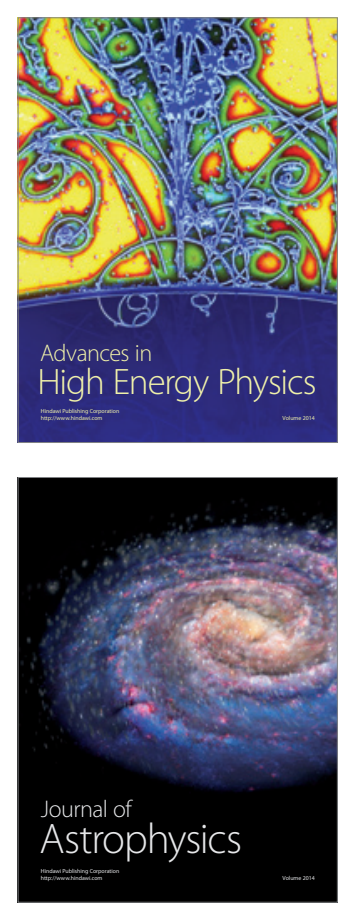
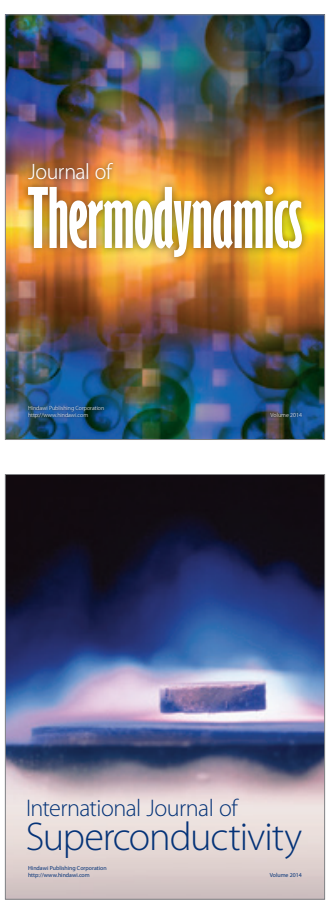
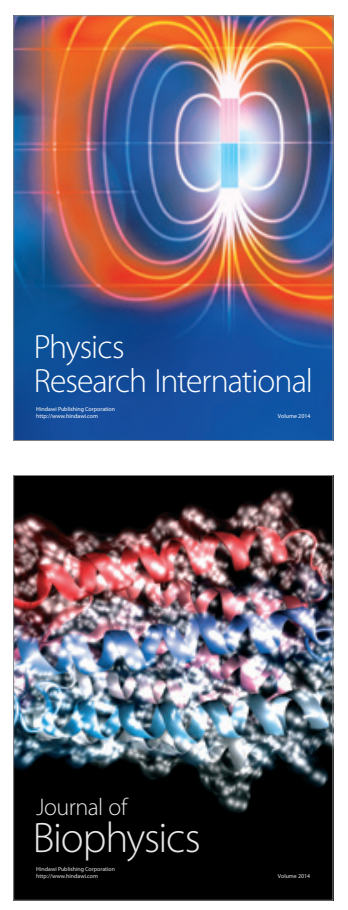
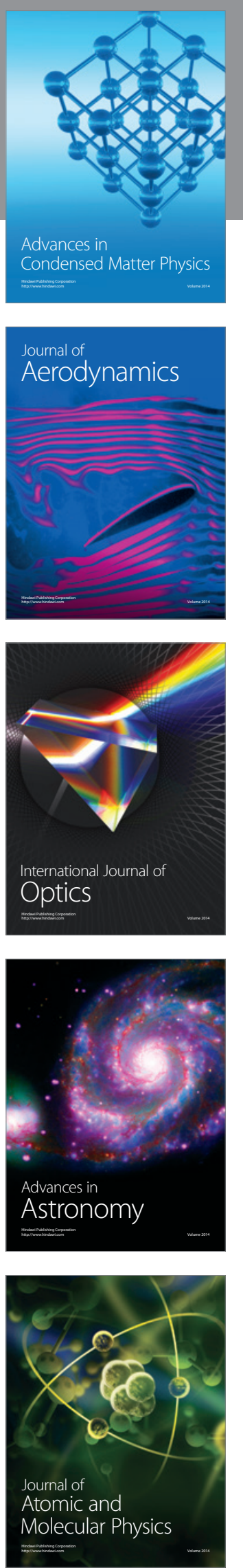